Original Research Article

\title{
Treatment of dermatophyte toenail onychomycosis with itraconazole
}

\author{
Vishal P. Giri ${ }^{1 *}$, Sudhir K. Gupta ${ }^{2}$, Om P. Giri ${ }^{3}$, Kirti V. ${ }^{1}$
}

${ }^{1}$ Department of Pharmacology,

Teerthanker Mahaveer Medical

College and Research Centre,

Moradabad, Uttar Pradesh, India

${ }^{2}$ Department of Skin and VD,

${ }^{3}$ Department of Pulmonary

Medicine, Darbhanga Medical

College and Hospital,

Darbhanga, Bihar, India

Received: 15 November 2016

Accepted: 09 December 2016

*Correspondence to:

Dr. Vishal P. Giri,

Email: drvpgiri@gmail.com

Copyright: (C) the author(s), publisher and licensee Medip Academy. This is an openaccess article distributed under the terms of the Creative Commons Attribution NonCommercial License, which permits unrestricted noncommercial use, distribution, and reproduction in any medium, provided the original work is properly cited.

\begin{abstract}
Background: Onychomycosis is fungal infection of nail. The prevalence of onychomycosis varies across the world. It is lower in tropical countries $(3.8 \%)$ than in sub-tropical countries and countries in the temperate zone $(23 \%)$. The risk of onychomycosis is higher in persons with diabetes mellitus and human immunodeficiency virus infection. Systemic antifungals are recommended for treatment. Topical antifungals are less effective. Different cure rates have been reported by different authors for terbinafine, itraconazole and fluconazole therapy. A variety of regimens (continuous or pulse) have also been suggested.

Methods: Here in this study we report a prospective, observational, cohort study of pulse itraconazole therapy in toenail onychomycosis caused by dermatophytes over a period of one year in patients with SCIO scores between 6 to 9 .

Results: It was observed that mean age of patients was 38.96 years. $57.27 \%$ study subjects had complete clinical improvement to oral itraconazole three pulses regimen. One pulse consisted of $200 \mathrm{mg}$ twice per day given for one week, followed by a three weeks interval. Thus, this regimen was repeated thrice.

Conclusions: This study shows the substantial benefit with itraconazole pulse regimen in toenail onychomycosis. The outcome this study is comparable with previously published data and may encourage the usage of itraconazole pulse regimen for the treatment of dermatophyte toenail onychomycosis in adults.
\end{abstract}

Keywords: Itraconazole, Onychomycosis, Pulse therapy

\section{INTRODUCTION}

Onychomycosis is the most common fungal infection of the nail bed, matrix and/or plate, representing up to $50 \%$ of all nail disorders. It causes discolouration, disfigurement and separation of nail from nail bed. Onychomycosis affects toenails more than fingernails because of their slower growth, reduced blood supply and frequent confinement in the dark, moist environment. Onychomycosis can be caused by dermatophytes, nondermatophyte molds (NDM) and candida species. Dermatophytes of genera Trichophoton and Epidermophyton are capable of invading nails. These breakdown and utilize keratin as a source of nitrogen. Dermatophytes responsible for onychomycosis are
Trichophoton rubrum, Trichophoton mentagrophytes, Trichophoton tonsurans, Trichophoton violaceum, Trichophoton schoenleinii, Trichophoton veruucusum and Epidermophyton floccosum. Nondermtophyte molds (fungi) include Aspergillus spp., Fusarium spp., Pennicillium spp., Acremonium spp., Alternaria spp., Clodosporium carrion and Geotrichum candicum. ${ }^{1,2}$

Accurate diagnosis of onychomycosis involves clinical and microscopic examination (for identification of fungal hyphae, pseudohyphae or spores) and culture (identification of organism). Histological evaluation using Periodic- Schiff staining increases the sensitivity for detecting the infection (indentify organism). Treatment is aimed at the eradication of the causative 
organism and return to normal appearance of the nail. Systemic antifungals are the most effective treatment. Topical therapy with ciclopiron nail lacquer, amorofine nail lacquer, and lacquer containing encecalin extract of Ageratina pichichenser, efinaconazole solution or tavaborole solution is less effective. To enhance the effectiveness of topical drugs attempts have been made to add penetration enhancer or incorporate the drug into transferome lipid vesicle. Surgical or chemical removal of infected nail parts is useful. It facilitates the penetration of antifungal drugs and eradicates reinfection sites so as to prevent recurrence and relapse. Laser therapy, photodynamic therapy, iontophoresis, ultrasound therapy, UVC radiation and psoralen with UVA (PUVA) show promise for onychomycosis treatment.,

Azoles group of oral antifungal drugs are used for the treatment of dermatophyte onychomycosis. Azoles are broadly classified into two groups - imidazoles and triazoles. They share the same antifungal spectrum and mechanism of action. The triazoles are metabolized more slowly and also have lesser effect on human sterol synthesis than imidazoles. The itraconazole, fluconazole, terconazole, voriconazole, posaconazole and isavuconazole are triazoles. The Imidazoles include clotrimazole, miconazole, ketoconazole, econazole, butoconazole, oxiconazole, sertaconazole and sulconazole. Terbinafine a synthetic allylamine is also effective in nail onychomycosis. 5,6

Azoles inhibit the growth of fungi by inhibiting a microsomal cytochrome P450 (CYP) enzyme 14-alphasterol demethylase. Inhibition of this enzyme impairs the biosynthesis of ergosterol for cytoplasmic membrane and leads to accumulation of 14-alpha-methyl-sterols which disrupt close packing of acyl chains of phospholipids thus impair the function of membrane-bound enzyme systems (ATPase and electron transport system enzymes). Azoles may also directly increase permeability of fungal cytoplasmic membrane.

Itraconazole is a synthetic triazole. It is an equimolar racemic mixture of four diastereo - isomers, each possessing three chiral centers. It is available in a capsule form. It is best absorbed in fed state. The half-life of itraconazole at a steady state is approximately 30-40 hours. It is metabolized in the liver. It is usually prescribed for toenail onychomycosis as $200 \mathrm{mg}$ once daily orally for three months - continuous therapy, or 200 mg twice daily orally for one week out of each month for three months- so called three pulse therapies. Retention of active drug in nail keratin permits for 28 weeks the intermittent treatment. Adverse drug reactions include nausea, vomiting, diarrhoea, abdominal discomfort, increased serum aminotranferase, increased serum triglycerides, hypokalemia, rash, hypertension, prolonged Q-T interval, ventricular dysfunction, congestive heart failure, lower limb edema, adrenal deficiency, rhabdomyolysis and stevens-johnson syndrome (rare).
The clinical types of onychomycosis of toenail are distal lateral subungual onychomycosis (DLSO), proximal subungual onychomycosis (PSO), superficial white onychomycosis (SWO), total dystrophic onychomycosis (TDO) and Enclonyx sublingual onychomycosos (ESO).

The aim of present study is to evaluate the effectiveness of itraconazole, three pulse therapies, for the treatment toenail onychomycosis caused by dermatophytes, in adults.

\section{METHODS}

The present prospective, observational study was conducted on patients who attended Out Patient Department (OPD) of the Department of Skin and VD, Darbhanga Medical College and Hospital, Darbhanga during period January 2015 to December 2015. One hundred seventy nine patients with clinical signs of persistent onychomycosis of toes were enrolled on for the study. Primary criteria for the clinical diagnosis of onychomycosis included white/yellow or orange/brown streaks or patches in or beneath the nail, and secondary criteria included lateral onycholysis, subungual hyperkeratosis and nail plate thickening. One hundred seventeen adults were included in the study group. The evaluation of disease severity was done on the basis of SCIO onycho-index and calculated by electronic calculator in the link http:www.onychoindex.com. Written consent was obtained from each patient. Drugs were branded and purchased by patients. The study was approved from institutional ethics committee.

\section{Inclusion criteria}

- $\quad$ Adults patients (age $>18$ years)

- Males and females (non-pregnant and non-lactating females)

- Clinical signs of onychomycosis of at least one great toe nail

- Positive microbiological evidence of fungal elements in nail specimen

- Positive culture for dermatophyte in nail specimen.

\section{Exclusion criteria}

- Females who are pregnant, lactating or likely to be pregnant

- Negative potassium hydroxide stain

- Negative mycological culture for dermatophytes consistent with onychomycosis

- Onychomycosis not caused by dermatophytes

- Immunocompromised either because of concomitant diseases e.g. HIV infection, or ongoing treatment e.g. chemotherapy

- Patients suffering from psoriasis, diabetes mellitus, renal failure, acute porphyria, ventricular dysfunction, congestive heart failure, abnormal liver function tests and haematological count abnormalities 
- Patients with history of serious liver disease, congestive heart failure, intake of oral antifungal drugs within 6 months prior to study, hypersensitivity to antifungal drugs

- Patients on concomitant therapy with drugs requiring P450 enzyme system.

\section{Procedures}

Nail specimen of each patient was collected from the toenail most affected by using an electric dental drill, after cleaning the area with $70 \%$ isopropyl alcohol to prevent contamination. The specimen was placed on a slide and a solution $20 \%$ potassium hydroxide and $40 \%$ dimethyl sulfoxide was dropped on the specimen. The prepared slide was heated for two to five minutes by an electric hot plate set at sixty to eighty degree Celsius. The specimen was then examined by a microscope under x100 magnification for the presence of fungal elements (hyaline septate hyphae and/or arthroconides). The nail specimen of each patient was also sent to the laboratory in a sterile container for culture of dermatophytes. The specimen was inoculated into three sets of test tubes first containing Sabouraud's dextrose agar (SDA) with $0.05 \%$ chloramphenicol, second Sabouraud's dextrose agar (SDA) with chloramphenicol and $0.5 \%$ cyclohexidine, and third Dermophyte test medium (DTM). Growth of dermatophytes in the media was observed regular once a week for 1 to 3 weeks. If no growth was detected after 3 weeks, specimen was considered as negative for dermatophytes. Dermatophyte species identification was done on the basis of characterstics colony morphology, growth rate (fast to very slow), characteristics microscopic features of various species of dermatophytes visible in the culture, supplemented by urease test, hair perforation test, slide culture and rice grain test. Medical data collected for each patient included age, gender, duration of onychomycosis, clinical types and SCIO score (scoring clinical index for onychomycosis score). SCIO score was calculated on the basis of three clinical factors

- Clinical form of onychomycosis (DLSO/ PSO/ $\mathrm{SWO} / \mathrm{TDO}$ ) which indicated location of fungus in nail

- Depth of nail involvement (onychomycosis localized to first one third / two third / more than two third nail plate length)

- Thickness of subungual hyperkeratosis (absent or nor more than one $\mathrm{mm} /$ one to two $\mathrm{mm} /$ more than two $\mathrm{mm})$.

SCIO score range from 1 to 30 and higher score indicates more severity. SCIO score 1 corresponds to SWO or marginal DLSO, while SCIO score 30 corresponds to whole nail DLSO with hyperkeratosis more than $2 \mathrm{~mm}^{7}$

\section{Treatment regimen}

Patients received a pulse regimen of itraconazole $(200 \mathrm{mg}$ twice daily) given after meals during the first week of each month, for three months. Patients were requested to visit OPD of the Department of Skin and VD, DMCH once a month for clinical check-up. At each visit clinical examination of nail and different systems of body was done.

Complete blood count, liver function tests (serum bilirubin, serum aspartate aminotransferase, serum alanine aminotransferase, serum alkaline phosphatase, and serum 5' nucleotidase and serum gamma-glutamyl transpeptidase), serum triglyceride, serum potassium, echocardiography and electrocardiography were carried out at the baseline and two weeks after the each three pulses. Mycological microscopy and cultures were performed at baseline and twelve months after the start of treatment. Effectiveness (clinical cure and mycological cure) was evaluated twelve months after the start of treatment.

\section{Primary outcome measure}

\section{Clinical cure}

Defined as $100 \%$ absence of clinical signs of onychomycosis, evident by normal appearing nail.

\section{Secondary outcome measure}

\section{Mycological cure}

Defined as absence of dermatophyte on microscopy and culture in nail specimen.

Patients were asked to report any adverse drug reaction (ADR) if they occur. Assessment of reported ADR for causality, severity and preventability were undertaken using Naranjo's causality, Hartwig and Siegel and Schumock and Thornton scales respectively. ${ }^{8-10}$

\section{Statistical analysis}

Statistical analysis was performed using SPSS version 20 for windows, (SPSS, Inc, Chicago, Illinois, United States of America). Data was expressed in Mean \pm standard deviation (SD).

\section{RESULTS}

One hundred seventeen adult patients suffering from dermatophyte onychomycosis of toenail were included in the study group. Seventy one were females and forty six males. Seventy four patients had distal lateral subungual onychomycosis (DLSO), thirty nine total dystrophic onychomycosis (TDO), two superficial white onychomycosis (SWO) and two proximal subungual onychomycosis (PSO). Trichophoton rubrum was isolated 
in seventy four cases, Trichophoton mentagrophytes in thirty one, Trichophoton tonsurans in six, Trichophoton violeceum in three and Trichophoton scholeinii in three cases. Mean age of study group patients was 38.96 years; mean duration of disease was 6.31 years (Table 1).

Table 1: Baseline characteristics of study subjects.

\begin{tabular}{|ll|}
\hline Characteristics & n $(\%)$ \\
\hline Gender & $46(39.32)$ \\
\hline Male & $71(60.68)$ \\
\hline Female & $74(63.25)$ \\
\hline Onychomycosis types & $39(33.33)$ \\
\hline $\begin{array}{l}\text { Distal lateral subungual } \\
\text { onychomycosis (DLSO) }\end{array}$ & $2(1.71)$ \\
\hline $\begin{array}{l}\text { Total dystrophic onychomycosis } \\
\text { (TDO) }\end{array}$ & $2(1.71)$ \\
\hline $\begin{array}{l}\text { Superficial white onychomycosis } \\
\text { (PWO) }\end{array}$ & $74(63.25)$ \\
\hline $\begin{array}{l}\text { Proximal subungual onychomycosis } \\
\text { (PSO) }\end{array}$ & $31(26.50)$ \\
\hline Dermatophytes & $6(5.13)$ \\
\hline Trichophoton rubrum & $3(2.56)$ \\
\hline Trichophoton mentagrophytes & $3(2.56)$ \\
\hline Trichophoton tonsurans & Mean (SD) \\
\hline Trichophoton violeceum & $38.96(12.13)$ \\
\hline Trichophton schoenleinii & $6.31(2.29)$ \\
\hline Characteristics & SD \\
\hline Age (year) & \\
\hline Duration of disease (year) & (2) \\
\hline
\end{tabular}

$\mathrm{n}=$ number of patients, $\%=$ percentage, $\mathrm{SD}=$ standard deviation.
Clinical cure was noted in sixty seven patients. Mycological cure was noted in seventy three patients. Three patients experienced nausea, two complained of vomiting and one experienced rash during first pulse therapy.

Table 2: Outcome variables.

\begin{tabular}{|ll|}
\hline Outcome & n (\%) \\
\hline Primary & \\
\hline Clinical cure achieved & $67(57.26)$ \\
\hline Clinical cure not achieved & $23(19.66)$ \\
\hline Secondary & \\
\hline Mycological cure achieved & $73(62.39)$ \\
\hline Mycological cure not achieved & $17(14.53)$ \\
\hline
\end{tabular}

$\mathrm{n}=$ number of patients, $\%=$ percentage.

Table 3: Evaluation of disease severity.

\begin{tabular}{|ll|}
\hline Duration & SCIO score mean ( SD ) \\
\hline At baseline & $7.79(0.89)$ \\
\hline $\begin{array}{l}3 \text { months after start of } \\
\text { treatment }\end{array}$ & $3.56(1.19)$ \\
\hline $\begin{array}{l}12 \text { months after start of } \\
\text { treatment }\end{array}$ & $0.51(1.23)$ \\
\hline
\end{tabular}

$\mathrm{SCIO}=$ Scoring clinical index for onychomycosis score, $\mathrm{SD}=$ Standard deviation.

They received symptomatic treatment, were improved but discontinued treatment. Abnormal liver function tests or haematological reports were not observed in any patient during follow up.

Table 4: Treatment response in onychomycosis with itraconazole pulse therapy.

\begin{tabular}{|c|c|c|c|c|c|}
\hline \multirow{2}{*}{$\begin{array}{l}\text { Onychomycosis } \\
\text { clinical types }\end{array}$} & \multirow{2}{*}{$\begin{array}{l}\text { At baseline } \\
\text { n }(\%)\end{array}$} & \multirow{2}{*}{$\begin{array}{l}\text { During } 1 \text { week } \\
\text { n }(\%)\end{array}$} & \multirow{2}{*}{$\begin{array}{l}\text { Follow up } 12 \\
\text { months n }(\%)\end{array}$} & \multicolumn{2}{|l|}{ Treatment response } \\
\hline & & & & Clinical cure n (\%) & Mycological cure n (\%) \\
\hline DLSO & $74(63.25)$ & $72(61.54)$ & $63(53.85)$ & $60(51.28)$ & $60(51.28)$ \\
\hline TDO & $39(33.33)$ & $36(30.77)$ & $24(20.51)$ & $4(3.42)$ & $10(8.55)$ \\
\hline SWO & $2(1.71)$ & $1(0.85)$ & $1(0.85)$ & $1(0.85)$ & $1(0.85)$ \\
\hline PSO & $2(1.71)$ & $2(1.71)$ & $2(1.71)$ & $2(1.71)$ & $2(1.71)$ \\
\hline Total & $117(100)$ & 111(98.87) & $90(76.92)$ & $67(57.26)$ & $73(62.39)$ \\
\hline
\end{tabular}

DLSO = Distal lateral subungual onychomycosis; TDO = Total dystrophic onychomycosis; SWO = Superficial white onychomycosis, $\mathrm{PSO}=$ Proximal subungual onychomycosis; At 1 week, six patients discontinued due to adverse drug reactions; 12 months after start of treatment. 21 patients were lost during follow up.

Table 5: Adverse drug reactions (ADR) observed during study.

\begin{tabular}{|lll|l|}
\hline ADR & $\begin{array}{l}\text { Casuality score } \\
\text { (Naranjo's scale) }\end{array}$ & $\begin{array}{l}\text { Severity grade (Hart wig and } \\
\text { Siegel scale) }\end{array}$ & $\begin{array}{l}\text { Preventability grade (Schumock and } \\
\text { Thornton scale) }\end{array}$ \\
\hline Nausea & 2 (Possible) & Moderate (Level 3) & Not preventable \\
\hline Nausea & 3 (Possible) & Mild (Level 2) & Not preventable \\
\hline Nausea & 3 (Possible) & Mild (Level 2) & Not preventable \\
\hline Vomiting & 2 (Possible) & Moderate (Level 3) & Not preventable \\
\hline Vomiting & 2 (Possible) & Mild (Level 1) & Not preventable \\
\hline Rash & 3 (Possible) & Mild (Level 1) & Not preventable \\
\hline
\end{tabular}




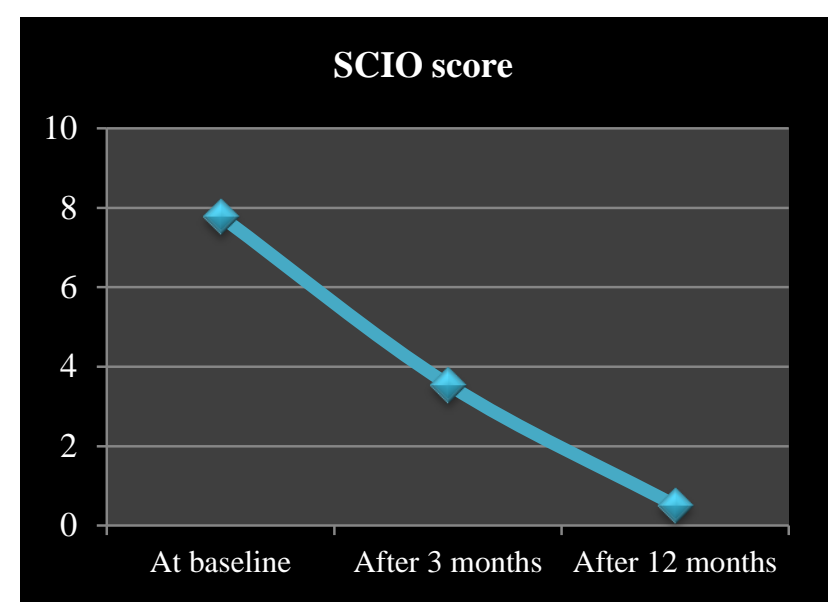

$\mathrm{SCIO}=$ Scoring clinical index for onychomycosis score .

Figure 1: Evaluation of disease severity.

Mean (SD) SCIO score decreased from 7.79 (0.89) at baseline to $0.51(1.23)$ twelve months after the start of treatment (Table 2), (Table 3), (Figure 1), (Table 4), (Table 5).

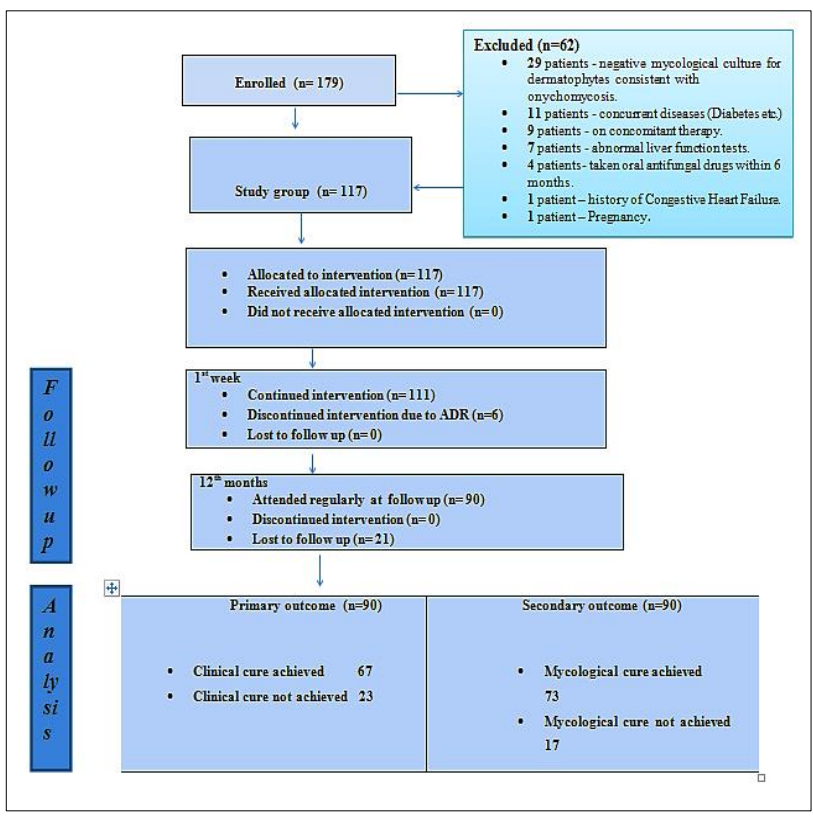

Figure 2: Flow chart of the cohort study participants.

Flow chart of the cohort study participants has been presented in (Figure 2).

\section{DISCUSSION}

The present study evaluated the effectiveness of itraconazole in dermatophyte toenail onychomycosis and achieved complete clinical cure rate of $57.26 \%$ and mycological cure rate of $62.39 \%$ by three pulses of oral itraconazole.

Ahmad et al reported clinical as well as mycological cure in $67.7 \%$ of patients treated with itraconazole pulse thearpy for dermatophyte toenail onychomycosis. ${ }^{11}$ Mishra et al noted clinical cure rate of $82 \%$ and mycological cure rate of $90 \%$ with itraconazole pulse therapy in dermatophyte caused toenail onychomycosis. ${ }^{12}$ Gupta et al attained mycological cure by $88.2 \%$ and clinical cure by $52.9 \%$ in patients of toenail onychomycosis caused by dermatophytes treated with itraconazole pulse therapy. ${ }^{13}$ They also observed itraconazole pulse therapy effective and safe in people with diabetes. ${ }^{14}$ Vora et al recorded cure rate of $40 \%$ with itraconazole in the treatment of onychomycosis. Coehlo et al. $^{15,16}$ reported itraconazole to be effective in onychomycosis, Pajaziti L and Vasiti observed mycological cure rate of $83.33 \%$ pulse regimen of itraconazole in onychomycosis. ${ }^{17}$

The present study observed lower clinical and mycological cure rates with pulse therapy regimen with itraconazole, than reported by many previous studies except one. The present study observation is similar to other studies as regards to the safety. Only $5.13 \%$ patients discontinued the study due to ADR. The only limitation of the present study is that long-term follow up has not been done to investigate for clinical and mycological recurrence.

\section{CONCLUSION}

Itraconazole oral pulse therapy is effective and safe and can be recommended for the treatment of dermatophyte toenail onychomycosis in adults. Further trials are required to evaluate number of pulse therapies required for dermatophyte toenail onchomycosis of different SCIO scores.

\section{ACKNOWLEDGEMENTS}

Authors would like to thanks all the participants of the study.

Funding: No funding sources

Conflict of interest: None declared

Ethical approval: The study was approved by the Institutional Ethics Committee

\section{REFERENCES}

1. Westerberg DP, Yoyack MJ. Onchomycosis: current trends in the diagnosis and management. Am Fam Physician. 2013;88:762-70.

2. Ranawaka RR, Nagahawatte, Gunasekara TA. Randomised, double-blind, comparative study on efficacy and safety of itraconazole pulse therapy and terbinafine pulse therapy on nondermatophyte mold onychomycosis: a study with 90 patients. J Dermatol Treat. 2016;27:364-72.

3. James Q, Del R. The role of topical antifungal therapy for onvhomycosis and emergence of new agents. J Clin Aesthet Dermatol. 2014;7(7):10-8. 
4. Bennett JE. Antifungal agents. In: Brunton LL, Chabner BA, Knollmann BC. eds. Goodman and Gillman's The Pharmacological Basis of Therapeutics. $12^{\text {th }}$ ed. New York, NY: McGraw-Hill; 2011:1571-1592.

5. Nenoff P, Paash U, Handrick W. Infectionn of finger and toenails due to fungi and bacteria. Hautarzt. 2014;65:337-46.

6. Takahata Y, Hiruma Y, Shiraki Y. Treatment of dermatpphyte onychomycosis with three pulses of terbinafine (500 mg per day for a week). Mycosis 2008;52:72-6.

7. Sergeev AY, Gupta AK, Sergeev VV. The scoring clinical index for onchomycosis. Skin Therapy Let. 2002;7:6-7.

8. Naranjo CA, Busto U, Sellers EM, Sandor P, Ruis I, Roberts EA, et al. A method for estimating the probability of adverse drug reactions. Clin Pharmacol Ther. 1981;30:239-45.

9. Hartwig SC, Siegel J, Schneider PJ. Preventability and severity assessment in reporting adverse drug reactions. Am J Hosp Pharm. 1992;49:2229-32.

10. Schumock GT, Thornton JP. Focusing on the preventability of adverse drug reactions. Hosp Pharm. 1992;27:538.

11. Ahmad A, Ali E, Khondker L, Ishamkhan MS. Pulse dose of oral itraconazole is effective in the treatment of onchomycosis. J Pak Asso Dermatol. 2011;21:276-80.
12. Mishra M, Panda P, Tripathy S. An open randomized comparative study of oral itraconazole pulse and terbinafine pulse in the treatment of onychomycosis. Indian J Dermatol Venerol Leprol. 2005;71:262-6.

13. Gupta AK, Paquet M, Simpson FC. Therapies for the treatment of onchomycosis. Clin Dermatol. 2013;31:544-54.

14. Gupta AK, Grover MD, Lynde CW. Pulse itraconazole versus continuous terbinafine for treatment of dermatophyte toenail onchomycosis in patients with diabetes mellitus. J Eur Acad Dermatol Venereol. 2006;20:1168-93.

15. Vora DN, Solanki P, Kothari A, Meher K. Study to compare efficacy of various antifungals (fluconazole, terbinafine, itraconazole) in the treatment of onychomycosis. J Res Med Den SCI. 2014;2:49-51.

16. Coehlo de sa D, Lamas APB, Tosti A. Drug therapy for onychomycosis: an evidence based review. Am J Clin Dermatol. 2014;15:17-36.

17. Pajaziti L, Vasiti E. Treatment of onychomycosis - A clinical study. Med Arh. 2015;69:173-6.

Cite this article as: Giri VP, Gupta SK, Giri OP, Kirti V. Treatment of dermatophyte toenail onychomycosis with itraconazole. Int J Basic Clin Pharmacol 2017;6:70-5. 